\title{
Large-eddy simulation of pollutant dispersion from a ground-level area source over urban street canyons with irreversible chemical reactions
}

\author{
T. Z. Du ${ }^{1,2}$, C.-H. Liu ${ }^{1}$, and Y. B. Zhao ${ }^{2}$ \\ ${ }^{1}$ Department of Mechanical Engineering, The University of Hong Kong, Hong Kong, China \\ ${ }^{2}$ Shenzhen Institute of Advanced Technology, Chinese Academy of Sciences, Shenzhen, China
}

Correspondence to: C.-H. Liu (liuchunho@graduate.hku.hk)

Received: 15 January 2014 - Revised: 15 September 2014 - Accepted: 23 September 2014 - Published: 23 October 2014

\begin{abstract}
In this study, the dispersion of chemically reactive pollutants is calculated by large-eddy simulation (LES) in a neutrally stratified urban canopy layer (UCL) over urban areas. As a pilot attempt, idealized street canyons of unity building-height-to-street-width (aspect) ratio are used. Nitric oxide (NO) is emitted from the ground surface of the first street canyon into the domain doped with ozone $\left(\mathrm{O}_{3}\right)$. In the absence of ultraviolet radiation, this irreversible chemistry produces nitrogen dioxide $\left(\mathrm{NO}_{2}\right)$, developing a reactive plume over the rough urban surface. A range of timescales of turbulence and chemistry are utilized to examine the mechanism of turbulent mixing and chemical reactions in the UCL. The Damköhler number $(D a)$ and the reaction rate $(r)$ are analyzed along the vertical direction on the plane normal to the prevailing flow at $10 \mathrm{~m}$ after the source. The maximum reaction rate peaks at an elevation where Damköhler number $D a$ is equal or close to unity. Hence, comparable timescales of turbulence and reaction could enhance the chemical reactions in the plume.
\end{abstract}

\section{Introduction}

Air pollutant dispersion in the UCL is mainly governed by air flows and turbulent mixing, which are indeed affected by both the atmospheric conditions and the rough urban surfaces at the bottom. Because most air pollutants are chemically reactive, the complicated reactions among different species cannot be neglected in air quality assessment. This paper thus attempts to shed some light on the relation between the turbulence and reactions in a plume.

\section{Methodology}

The computational domain $(L=72 \mathrm{~m}, W=5 \mathrm{~m}, H=8 \mathrm{~m})$ consists of 36 repeated street canyons placed perpendicular to the prevailing flow (Fig. 1). The street canyon aspect ratio equals unity $(h=w=1 \mathrm{~m})$ so the flows fall into the skimming flow regime (Oke, 1988). The spatial domain is discretized into 15 million hexahedral elements. The first grid point is located less than 6 wall units from the nearby solid boundaries.
The UCL flow is assumed to be incompressible and isothermal. The LES is used instead of the Reynoldsaveraged Navier-Stokes turbulence models because of its accuracy (Tominaga and Stathopoulos, 2011). The resolvedscale Navier-Stokes equations are

$\frac{\partial \bar{u}_{i}}{\partial t}+\frac{\partial \bar{u}_{i} \bar{u}_{j}}{\partial x_{j}}=-\frac{\partial \bar{\pi}}{\partial x_{i}}+v \frac{\partial^{2} \bar{u}_{i}}{\partial x_{j} \partial x_{j}}-\frac{\partial \tau_{i j}^{\mathrm{a}}}{\partial x_{j}}-\Delta P_{x} \delta_{i 1}$

where $\bar{\pi}\left(=\bar{p} / \rho+\frac{2}{3} k_{\mathrm{SGS}}\right)$ is the modified pressure with the subgrid-scale (SGS) kinetic energy $k_{\mathrm{SGS}}$, and $\tau_{i j}^{\mathrm{a}}\left(=-2 v_{\mathrm{SGS}} \bar{S}_{i j}\right)$ is the anisotropic SGS stress tensor with the SGS eddy viscosity $v_{\mathrm{SGS}}\left(=C_{k} k_{\mathrm{SGS}}{ }^{1 / 2} \Delta\right)$, in which $C_{k}(=0.07)$ is an empirical constant and $\Delta\left(=V_{\text {cell }}^{1 / 3}\right)$ is the filter size. The flow is driven by the pressure gradient $\Delta P_{x}$ $\left(=0.0004 \mathrm{~m} \mathrm{~s}^{-2}\right)$ so the freestream velocity $u_{\infty}$ is close to unity. The kinematic viscosity $v$ equals $0.0001 \mathrm{~m}^{2} \mathrm{~s}^{-1}$, hence, the Reynolds number $\operatorname{Re}\left(=u_{\infty} h / v\right)=11570$ which is high enough for turbulent flows. Based on the friction 


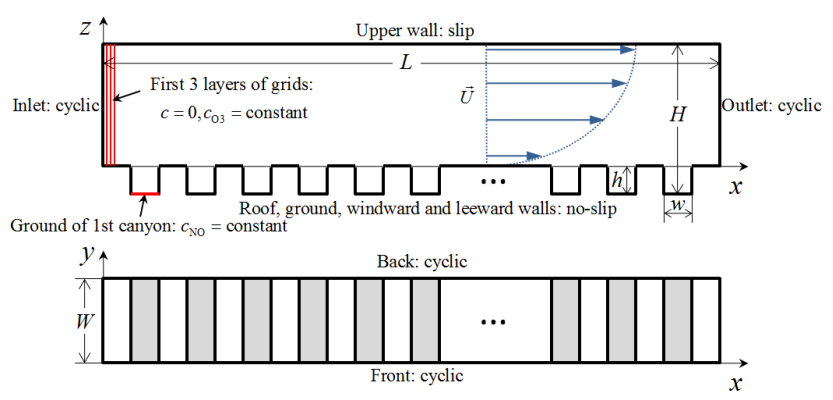

Figure 1. Computational domain and boundary conditions.

velocity $u_{\tau}$, the friction Reynold number $\operatorname{Re}_{\tau}\left(=u_{\tau} h / v\right)$ equals 529.

The closure term $\tau_{i j}^{\mathrm{a}}$ is modeled by the SGS $k$-equation eddy-viscosity model

$$
\begin{aligned}
\frac{\partial k_{\mathrm{SGS}}}{\partial t} & +\frac{\partial \bar{u}_{j} k_{\mathrm{SGS}}}{\partial x_{j}}=-\tau_{i j}^{\mathrm{a}} \frac{\partial \bar{u}_{i}}{\partial x_{j}}+\frac{\partial}{\partial x_{j}}\left(v_{\mathrm{eff}} \frac{\partial k_{\mathrm{SGS}}}{\partial x_{j}}\right) \\
& -C_{\varepsilon} \frac{k_{\mathrm{SGS}}^{3 / 2}}{\Delta}
\end{aligned}
$$

where $v_{\text {eff }}\left(=v+v_{\mathrm{SGS}}\right)$ is the effective viscosity and $C_{\varepsilon}(=1.05)$ is another empirical constant.

The chemical reaction considered is the irreversible $\mathrm{O}_{3}$ titration

$\mathrm{NO}+\mathrm{O}_{3} \stackrel{k_{3}}{\longrightarrow} \mathrm{NO}_{2}+\mathrm{O}_{2}$

where $k_{3}\left(=44.05 e^{-1370 / T} \mathrm{ppm}^{-1} \mathrm{~s}^{-1}\right)$ is the temperature $T(=298.15 \mathrm{~K})$ dependent reaction rate constant.

The concentrations of chemical species are calcuated by the advection-diffusion equations

$$
\begin{aligned}
& \frac{\partial \bar{c}_{\mathrm{NO}}}{\partial t}+\frac{\partial \bar{c}_{\mathrm{NO}} \bar{u}_{j}}{\partial x_{j}}-\left(\frac{v}{S c}+\frac{\nu_{\mathrm{SGS}}}{S c_{t}}\right) \frac{\partial^{2} \bar{c}_{\mathrm{NO}}}{\partial x_{j} \partial x_{j}} \\
& +k_{3} \bar{c}_{\mathrm{NO}} \bar{c}_{\mathrm{O}_{3}}=0 \\
& \frac{\partial \bar{c}_{\mathrm{O}_{3}}}{\partial t}+\frac{\partial \bar{c}_{\mathrm{O}_{3}} \bar{u}_{j}}{\partial x_{j}}-\left(\frac{v}{S c}+\frac{\nu_{\mathrm{SGS}}}{S c_{t}}\right) \frac{\partial^{2} \bar{c}_{\mathrm{O}_{3}}}{\partial x_{j} \partial x_{j}} \\
& +k_{3} \bar{c}_{\mathrm{NO}} \bar{c}_{\mathrm{O}_{3}}=0
\end{aligned}
$$

where $S c(=0.72)$ is the Schmidt number, $S c_{t}(=0.72)$ is the turbulent Schmidt number.

The time derivatives are discretized by the second-order backward differencing, the gradient terms of pressure and velocity are discretized by the central differencing and the convection terms are discretized by the total variation diminishing (TVD) scheme. In the diffusion terms, the central differencing and the explicit non-orthogonal correction are separately used for the approximation schemes of the diffusion coefficients and the surface normal gradients. The LES model was validated by the experimental data of Builtjes (1983).

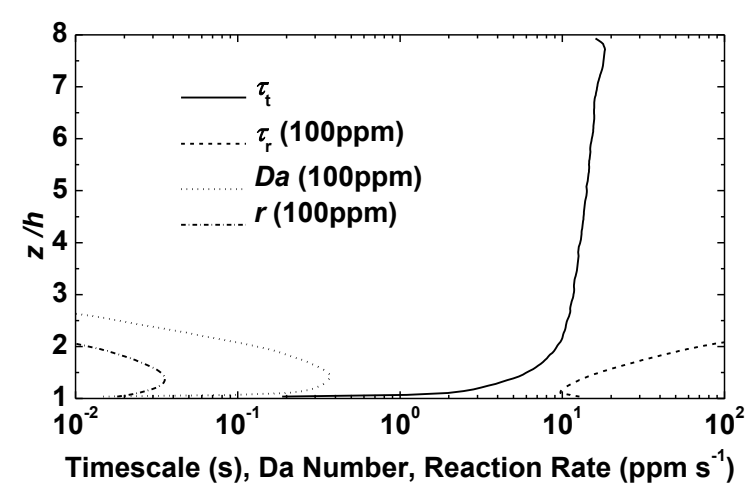

(a) $C_{\mathrm{NO}}=100 \mathrm{ppm}, X=10 \mathrm{~m}$

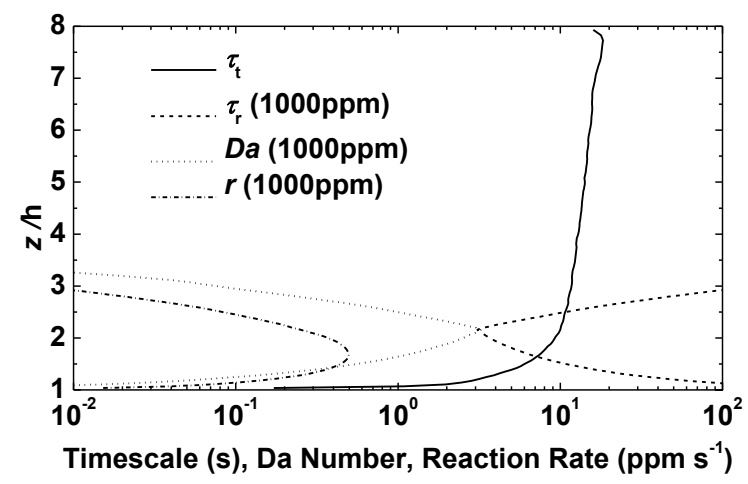

(b) $C_{\mathrm{NO}}=1000 \mathrm{ppm}, X=10 \mathrm{~m}$

Figure 2. Vertical profiles of turbulent timescale $\tau_{\mathrm{t}}$, reaction timescale $\tau_{\mathrm{r}}$, Damköhler number $D a$ and reaction rate $r$ (averaged in the spanwise direction).

The roof, ground, windward and leeward walls are no-slip boundaries. The upper wall is slip. The inlet and outlet are cyclic so the flow circulates from the outlet to the inlet. The front and back surfaces are cyclic so the spanwise domain is infinite. The NO concentration on the ground of the first street canyon is constant $(1,10,100$ or $1000 \mathrm{ppm})$ in each configuration. In the first 3 layers of grids after the inlet, the $\mathrm{O}_{3}$ concentration is kept constant $(1 \mathrm{ppm})$ while the concentrations of the other species ( $\mathrm{NO}, \mathrm{NO}_{2}$ and $\mathrm{O}_{2}$ ) are equal to zero in order to prevent the species at the outlet from periodically re-entering the spatial domain.

The time step increment is $0.01 \mathrm{~s}$ so that the Courant number $(\approx 0.3)$ is less than 1 . The initial calculation needs more than $1000 \mathrm{~s}$ for fully developed flows. The time for statisitcs collection after the initial spinning up is $400 \mathrm{~s}$ and the time interval for data sampling is $0.1 \mathrm{~s}$. 


\section{Timescale analysis}

The total lifetime is the ratio of turbulent kinetic energy and dissipation rate. The dissipation lifetime is a tenth of the total lifetime (Pope, 2000). Therefore, the turbulence timescale is

$\tau_{\mathrm{t}} \approx 0.1 \frac{k_{\mathrm{t}}}{\left\langle\varepsilon_{\mathrm{f}}\right\rangle}$

where $k_{\mathrm{t}}\left(=\frac{1}{2}\left\langle u_{i}^{\prime \prime} u_{i}^{\prime \prime}\right\rangle\right)$ is the turbulent kinetic energy and $\varepsilon_{\mathrm{f}}\left(=v \frac{\partial \bar{u}_{i}}{\partial x_{j}} \frac{\partial \bar{u}_{i}}{\partial x_{j}}\right)$ is the dissipation rate of the filtered kinetic energy (Pope, 2000). Here $\left\langle\varepsilon_{\mathrm{f}}\right\rangle$ represents the spatio-temporal average of the filtered dissipation rate.

Because reaction rates are determined by the slowest one, the reaction timescale is equal to the largest timescale between $\mathrm{NO}$ and $\mathrm{O}_{3}$

$\tau_{\mathrm{r}}=\max \left(\tau_{\mathrm{NO}}, \tau_{\mathrm{O}_{3}}\right)$

where $\tau_{\mathrm{NO}}=\frac{1}{k_{3}<\bar{c}_{O_{3}}>}$ and $\tau_{\mathrm{O}_{3}}=\frac{1}{k_{3}<\bar{c}_{N O}>}$, are the reaction timescales of $\mathrm{NO}$ and $\mathrm{O}_{3}$, respectively.

The chemical reaction mechanism is measured by the dimensionless Damköhler number

$D a=\tau_{\mathrm{t}} / \tau_{\mathrm{r}}$

That is the ratio of turbulence timescale to reaction timescale. When $D a>1$, the chemical reaction is faster than the turbulent mixing. When $D a<1$, the turbulent mixing is stronger than the chemical reaction (Meeder and Nieuwstadt, 2000).

When the maximum $D a$ is less than 1 (Fig. 2a), the reaction rate $r\left(=k_{3}\left\langle\bar{c}_{\mathrm{NO}}\right\rangle\left\langle\bar{c}_{\mathrm{O}_{3}}\right\rangle\right)$ peaks at the height where $D a$ reaches the maximum. The maximum $D a$ is at the location where the turbulence timescale is closest to the reaction timescale, implying that comparable timescales of turbulence and reaction are crucial to the reaction rate.

When the maximum $D a$ is larger than 1 (Fig. 2b), there are two points at which $D a=1$. The height of the maximum reaction rate is very close to that of the lower $D a=1$ point where the timescales of turbulence and reaction are comparable to each other. The upper $D a=1$ point also has comparable timescales, however, its reaction rate is delayed by the weaker turbulent mixing (longer turbulence timescale).

\section{Conclusions}

Pollutant plume dispersion with irreversible chemistry over idealized urban roughness is performed by LES. With increasing wall-normal distance from the bottom roughness elements, the turbulence timescale increases. On the contrary, the reaction timescale decreases initially then increases quickly. The height of maximum reaction rate is observed where $D a$ number is equal or close to unity, implying that comparable timescales of turbulence and reaction is one of the factors enhancing chemical reactions.

Acknowledgements. This project is partly supported by HK RGC GRF HKU 714412E. Technical support from Lilian Y. L. Chan, W. K. Kwan and N. S. Mok with ITS, HKU is appreciated.

Edited by: M. Piringer

Reviewed by: K. Baumann-Stanzer and two anonymous referees

\section{References}

Builtjes, P. J. H.: A comparison between chemically reacting plume models and wind tunnel experiments, in: Air Pollution Modeling and its Application II, edited by: Wispelaere, C.,, Plenum Press, New York, 59-83, 1983.

Meeder, J. P. and Nieuwstadt, F. T. M.: Large-eddy simulation of the turbulent dispersion of a reactive plume from a point source into a neutral atmospheric boundary layer, Atmos. Environ., 34, 3563-3573, 2000.

Oke, T. R.: Street design and urban canopy layer climate, Energy Build., 11, 103-113, 1988.

Pope, S. B.: Turbulent Flows, Cambridge University Press, Cambridge, 2000.

Tominaga, Y. and Stathopoulos, T.: CFD modeling of pollution dispersion in a street canyon: Comparison between LES and RANS, J. Wind Eng Ind. Aerodyn., 99, 340-348, 2011. 